\title{
LOS PERFILES INTERPERSONALES: ASPECTOS CLÍNICOS DEL CIRCUMPLEX INTERPERSONAL DE WIGGINS
}

\author{
ELENA FELIPE ${ }^{1}$ y ALEJANDRO ÁVILA ${ }^{2}$ \\ ${ }^{1}$ Universidad de Extremadura \\ ${ }^{2}$ Universidad de Salamanca
}

(Aceptado en octubre de 2001)

\begin{abstract}
Las Escalas de Adjetivos Interpersonales [IAS; Wiggins, 1979, Wiggins, Trapnell y Phillips, 1988. Adaptación al castellano por A. Ávila (Wiggins, 1996)] evalúan el estilo interpersonal de la personalidad normal con una fuerte estructura conceptual que deriva de importantes tradiciones de investigación en Psicología. El circumplex interpersonal de Wiggins y las IAS han demostrado una importante utilidad clínica, tanto en el diagnóstico como en la intervención. Este estudio examina las propiedades clínicas de las IAS mediante el análísis de las diferencias en el estilo interpersonal entre sujetos clínicos y no clínicos junto con el análisis de los perfiles interpersonales y su significado clínico. Encontramos diferencias importantes en el estilo interpersonal entre ambos grupos de sujetos, así como respecto al significado clínico-diagnóstico de los perfiles interpersonales obtenidos con las IAS.
\end{abstract}

Palabras clave: Conducta interpersonal, Escalas de Adjetivos Interpersonales, circumplex interpersonal.

\section{Interpersonal profiles: Clinical aspects of Wiggins's interpersonal circumplex}

The Interpersonal Adjective Scales [IAS; Wiggins, 1979; Wiggins, Trapnell and Phillips, 1988. Spanish version by A. Ávila (Wiggins, 1996)] assess interpersonal profiles of normal personality and has a strong conceptual structure which draws from relevant tradition in psychological research. Wiggins's interpersonal circumplex and IAS have demonstrated an important clinical value, both in diagnostic and intervention. This study examined the IAS clinical properties, by means of analysis of interpersonal style differences between clinical and no clinical participants. We also analysed the interpersonal profiles and clinical significance. We found significant differences between clinical and no clinical participants on interpersonal style and clinical-diagnostic meaning of IAS interpersonal profiles.

Key words: Interpersonal behavior, Interpersonal Adjetives Scales, IAS, interpersonal circumplex.

\section{INTRODUCCIÓN}

Si queremos describir o explicar nuestro comportamiento no podemos dejar de lado el estudio de las relaciones que establecemos con otras personas, de nuestra conducta interpersonal. Todas nuestras conductas tienen un componente interpersonal, van dirigidas hacia otra persona o la implican de una $u$ otra forma y su

Correspondencia: Elena Felipe, E.U. de Terapia Ocupacional, Campus Universitario, Av. de la Universidad, s/n, 10071 Cáceres. Correo-e: efelipe@unex.es importancia para nuestra supervivencia y desarrollo se manifiesta ya desde nuestro nacimiento, en los vínculos que establecemos con las figuras tempranas de apego y que serán decisivos para la formación de nuestra personalidad.

El estudio científico de la conducta interpersonal en psicología la llevan a cabo dos disciplinas complementarias: la psicología social y la psicología de la personalidad. Ambas comparten el objetivo, intentar explicar las razones de nuestro comportamiento, pero lo hacen desde puntos de vista y con métodos y 
técnicas de investigación diferentes. Desde la psicología de la personalidad, uno de los primeros teóricos en dedicarse al estudio de la conducta interpersonal fue Leary (1957), quien la definió como toda conducta que está relacionada abierta, consciente, ética o simbólicamente con otro ser humano real, colectivo o imaginado. Leary inicia así un prolífico campo de investigación a partir del cual se desarrollaron modelos e instrumentos de evaluación de la personalidad con características propias, como la metodología circumplex, y técnicas de intervención clínica, que continúan utilizándose en nuestros días.

De todos los modelos interpersonales uno de los mas importantes y de mayor repercusión dentro de la psicología de la personalidad es el circumplex interpersonal de Jerry S. Wiggins y su cuestionario asociado: las Escalas de Adjetivos Interpersonales (Interpersonal Adjectives Scales; IAS). Wiggins construyó estas escalas a partir de la concepción neo-sulliviana de la cognición social que enfatiza el intercambio de afecto positivo (amor) y estatus en las transacciones interpersonales (Carson, 1969; Foa y Foa, 1974; Leary, 1957; Sullivan, 1953) y se apoyó en una fuerte estructura conceptual originada en cinco tradiciones de investigación, algunas de las cuales cuentan con una historia de más de 50 años y en campos tan diversos como la teoría y evaluación de la personalidad, la psicología clínica y la psiquiatría, la sociología y la psicología social y la psicometría. Repasaremos brevemente cada una de ellas.

La tradición léxica en personalidad parte de la premisa de que las diferencias individuales más sobresalientes y socialmente relevantes en la vida de las personas han quedado codificadas en sus lenguajes naturales (Goldberg, 1982). Esta tradición comienza en 1936 con la recopilación de Allport y Odbert de los términos descriptivos de los rasgos a partir del diccionario Webster, que posteriormente repitieron Norman (1967) y Goldberg (1977). Para la construcción de las IAS, Wiggins (1979) se basó en el trabajo de este último autor.

La tradición teórica interpersonal en psicología clínica y psiquiatría (Kiesler, 1982) se inició con la teoría interpersonal de la personalidad de H.S. Sullivan. Este autor, junto con otros teóricos como Horney y Fromm, concedía mayor importancia a los aspectos sociales en sus teorías de la personalidad diferenciándose así de las corrientes teóricas de ese momento. Esta tradición fue convertida en procedimientos de evaluación de la personalidad por Freedman, Leary, Ossorio y Coffey (1951) y elaboradas en un contexto clínico por Leary (1957). Este grupo de investigadores, llamado grupo Kaiser, realizó numerosas innovaciones conceptuales y técnicas, de las que podemos destacar como fundamental para el desarrollo de las IAS, el descubrimiento de que las variables interpersonales dibujan un orden circular alrededor de dos coordenadas ortogonales de poder y afiliación.

Posteriormente al grupo Kaiser, Guttman (1954) descubre el ordenamiento circumplex entre las medidas psicológicas, iniciando así la tradición del análisis ordinal y de facetas. Guttman desarrolló el circulo de variables para el estudio de la inteligencia, mediante una estructura bidimensional, Radex, que representa las interrelaciones entre las puntuaciones de test de inteligencia. Observó que cuando dos test medían la misma variable con diferente intensidad pero al mismo nivel de complejidad, las correlaciones se agrupaban en una única faceta o factor, a esta estructura la denominó Simplex, y es una dimensión vertical de complejidad. Cuando diferían en clase de inteligencia, las correla- 
ciones configuraban varias facetas. En este caso, Guttman propuso que si se ordenaran espacialmente los coeficientes de correlación, según su tamaño, formarían un círculo, y que la matriz de correlaciones se ordenaría de forma que las correlaciones disminuirían al descender en columnas y filas y se incrementarían a partir de la diagonal. Esta segunda dimensión, denominada circumplex, describe el orden entre test de diferente contenido y la misma complejidad. En resumen, partiendo de la matriz de correlaciones obtenidas de las variables de interés, Guttman obtiene mediante el análisis de facetas, los factores o variables comunes a dichas variables y a partir de ellos propone un ordenamiento que permite medir e interpretar los datos interpersonales según los modelos interpersonales de evaluación.

Las tradiciones del intercambio social y las tendencias de gestión y dirección proceden tanto de la sociología como de la psicología social (Carson, 1969) y fueron originadas en 1959 por Goffman. En la actualidad están representadas por autores como Cheek y Hogan (1982) y su importancia se manifiesta en las formulaciones respecto a la complementariedad en las relaciones interpersonales propuesta por Wiggins $(1980,1982)$ en las que la idea central es que el actor negocia su definición preferida de la situación interpersonal en términos de amor y estatus.

Por último la tradición multivariada se inicia con el desarrollo por parte de Thurstone de los métodos de análisis factorial, y llega hasta el modelo de los Cinco Factores que junto con el modelo circumplex de la conducta interpersonal, dominan la actual escena en la estructura de personalidad (Wiggins y Pincus, 1992). Wiggins, Trapnell y Phillips (1988) y Pincus y Wiggins (1990) han demostrado que los dos modelos son complementarios y que pueden utilizarse conjuntamente.

Asentadas en estas cinco tradiciones de investigación las Escalas de Adjetivos Interpersonales originales fueron publicadas en 1979 por Wiggins. Posteriormente en 1988 apareció una versión revisada de las IAS (IAS-R, Wiggins, Trapnell y Phillips) y en 1990 las IASR-B5 (Trapnell y Wiggins), que son una versión de las Escalas IAS construidas para cubrir íntegramente el universo de contenido del modelo de los cinco factores de personalidad. En 1996 se publicó la adaptación y validación a la población española de las Escalas de Adjetivos Interpersonales realizada por Alejandro Ávila-Espada (también denominadas IAS) que mantienen todas las características del instrumento original, y que es la que vamos a utilizar en este trabajo. Las IAS son una lista de 64 adjetivos que describen las interacciones interpersonales mediante una escala tipo Likert de 8 intervalos en el que la persona debe responder la exactitud con la que cada adjetivo le describe en sus relaciones interpersonales. Evalúan tanto el tipo interpersonal como su intensidad y proporcionan una representación del perfil interpersonal utilizando los valores de dominancia (DOM) y sostenimiento (LOV) como ejes ortogonales a partir de los cuales el perfil se dibuja con las puntuaciones en los ocho octantes (Wiggins, 1996). En la Figura 1 podemos ver un perfil obtenido con las IAS, vemos a la derecha de la figura superior las puntuaciones directas y las puntuaciones $\mathrm{T}$ que obtienen en cada uno de los octantes (en la Tabla 1 podemos ver el nombre de cada uno de los octantes IAS) y la representación del perfil interpersonal, y en la figura inferior vemos el gráfico polar con las puntuaciones DOM y LOV y el vector junto con la puntuación de Longitud del vector (LongV) que establecen el tipo interpersonal, en ese caso $\mathrm{DE}$ (frío de ánimo). 


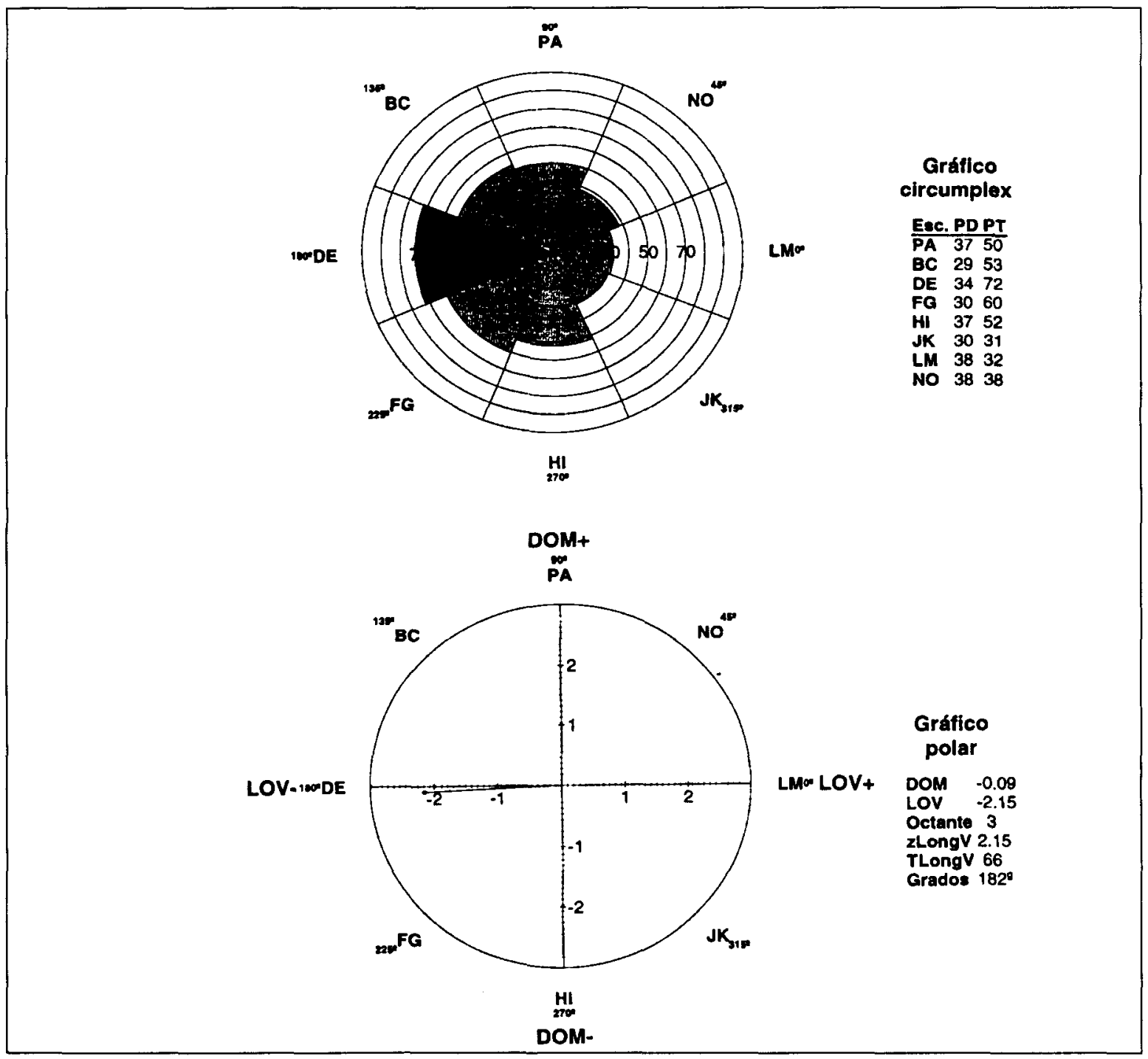

Figura 1. Perfil circumplex regular y gráfico polar obtenido con las IAS

Estas escalas se utilizan principalmente para la evaluación psicológica y clínica del estilo de conducta interpersonal. También son utilizadas como instrumento de investigación en psicología de la personalidad y psicología clínica, sirviendo como marcadores semánticos del espacio interpersonal para propósitos de comparación con otros sistemas de evaluación, y para identificar dimensiones de conducta interpersonal medidas por escalas empleadas en otras investigaciones experimentales de personalidad (Wiggins, 1980). Desde su publicación en
1979 las IAS han sido utilizadas en numerosos contextos de investigación, utilizando las diferentes posibilidades que las escalas ofrecen: su estructura conceptual; las escalas u octantes y los ítems. Su estructura conceptual se ha empleado entre otros en la identificación de constructos disposicionales (Buss y Craik, 1985) y en la definición de la estructura de situaciones interpersonales (Kelley, 1983). La estructura circumplex ofrecida por el IAS ha sido también relacionada con estructuras circumplex similares y otros sistemas de clasificación como las 
necesidades de Murray (p.ej., Wiggins y Broughton, 1991), los problemas interpersonales (p.ej., Alden, Wiggins y Pincus, 1990) y los trastornos de personalidad (p.ej., Felipe y Ávila, en prensa, Wiggins, 1982, Wiggins y Pincus, 1989).

Las escalas se han empleado en estudios sobre atribución de rasgos a estudiantes con discapacidad (Fichten y Amsel, 1986) y en el estudio del autoconcepto de personas con lesiones medulares traumáticas y su relación con síntomas depresivos e integración social (Felipe y Ávila, 1999).

El conjunto de ítems de las IAS originales sirve como estímulo en estudios experimentales de estereotipos personales (Beringer y DeSoto, 1985) y en la verificación de rasgos de personalidad (Romer y Revelle, 1985).

Finalmente, en el ámbito clínico, las IAS se han utilizado entre otros en la evaluación del estilo de relaciones interpersonales y la elaboración de los objetivos de la intervención en personas con trastornos de la alimentación (Madison, 1997), y en el estudio de los efectos de la personalidad del cliente en su presentación interpersonal durante la terapia racional emotiva y su relación con el proceso psicoterapéutico (Dempsey, Lamon, Suderland y DiGiuseppe, 1994).
Dentro del ámbito clínico un área aún poco estudiada y que puede tener gran importancia para el diagnóstico y la intervención, es la utilidad y aplicación de los perfiles interpersonales, especialmente los aspectos relacionados con la adecuación a la estructura correlacional que deben mantener según el modelo estructural interpersonal, así como la bipolaridad de las Escalas, lo que Wiggins y Trapnell denominan "la nave espacial interpersonal» (1996, p. 127), según la cual el sujeto presenta una puntuación más elevada en uno de los octantes IAS y las puntuaciones de los octantes adyacentes se irán reduciendo hasta llegar al octante opuesto que obtendrá la menor puntuación. Hay casos en los que no se cumple esta estructura de puntuaciones y obtenemos perfiles, que denominaremos como irregulares, en los que puede haber simultáneamente puntuaciones elevadas en octantes alejados e incluso opuestos. En el perfil superior de la Figura 1 podemos ver un ejemplo de un perfil regular y en la Figura 2 un ejemplo de un perfil irregular. En general, para Wiggins «los perfiles que se apartan de forma acentuada de la configuración esperada deberían ser vistos con sospecha y recelo" (1996, p. 27), aunque no especifica a que hace referencia esa sospecha.
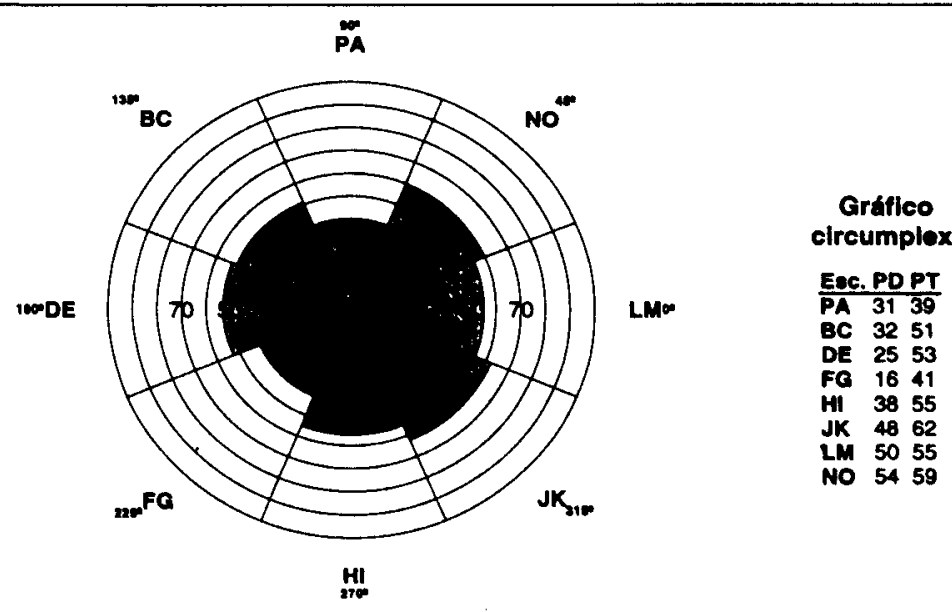

Figura 2. Perfil circumplex irregular obtenido con las IAS 
Con este trabajo pretendemos explorar la utilidad en contextos clínicos de las IAS, en concreto si existen diferencias en el estilo de conducta interpersonal entre sujetos clínicos y no clínicos y en que octantes y puntuaciones se manifiestan esas diferencias, haciendo especial insistencia en la puntuación de longitud del vector (LongV) que en otros trabajos ha sido relacionada con características psicopatológicas (Wiggins, Trapnell y Phillips, 1988). Además, dentro de los sujetos clínicos, vemos si existen diferencias psicopatológicas entre aquellos sujetos que presentan perfiles regulares e irregulares, es decir, entre aquellos sujetos cuyos perfiles cumplen el modelo estructural propuesto por Wiggins y aquellos que no lo cumplen.

\section{MÉTODO}

\section{Instrumentos de medida}

Escalas de Adjetivos Interpersonales (IAS) (Wiggins, 1996, adaptación española de A. Ávila).

Inventario Clínico Multiaxial de Millon (MCMI-II; Millon, 1987, 1998). Cuestionario de personalidad que proporciona información referente a tareas de evaluación y toma de decisiones de tratamiento sobre personas con dificultades emocionales e interpersonales. Este cuestionario, que debe utilizarse siempre con población clínica, tiene 175 ítems, con formato de respuesta verdadero/falso. Está compuesto por 26 escalas, cuatro de validez y fiabilidad y 22 escalas clínicas, en concreto: 10 escalas básicas (patrones básicos) de personalidad, tres escalas de trastornos de personalidad patológicos y nueve escalas de síndromes clínicos (Eje I del DSM-III-R).

Inventario de Síntomas SCL-90-R (Derogatis, 1983; González de la Rivera et al., 1989). Autoinforme clínico que evalúa la conducta sintomática y el malestar psicológico de pacientes ambulatorios de salud mental. Esta formado por 90 ítems con formato de respuesta es una escala tipo Likert de cinco intervalos, que va desde 0 (nada) a 4 (extremadamente), a la que la persona ha de responder hasta que punto se ha sentido molesto por el síntoma que plantea el ítem en un periodo que abarca desde la última semana hasta el día de la evaluación. Las dimensiones sintomáticas que mide el SCL-90-R son las siguientes: Somatización (SOM), Obsesivocompulsiva (OBS), Sensibilidad interpersonal (SEN), Depresión (DEP), Ansiedad (ANS), Hostilidad-Miedo (HOS), Ansiedad fóbica (FOB), Ideación paranoide (PAR) y Psicoticismo (PSI). Además de estas dimensiones, el SCL90-R proporciona tres índices globales que evalúan el nivel o profundidad de la psicopatología del sujeto y nos ayudan a apreciar mejor el cuadro clínico que presenta. Estos índices son: Índice global de gravedad (IGS); Índice de malestar (PSDI) y Total de síntomas positivos (TSP).

\section{Participantes}

Para este trabajo hemos utilizado dos grupos de participantes: (a) Un grupo de sujetos no clínico $(n=140)$, formado por estudiantes universitarios heterogéneos. De ellos $66(47,2 \%)$ eran varones y 74 $(52,8 \%)$ mujeres. La media de edad es de 22,3 años (desviación típica: 3,36). Las puntuaciones de los participantes se obtuvieron a través de la administración de los cuestionarios como parte de una tarea práctica en una asignatura de introducción a la psicología. (b) Un grupo de sujetos clínicos $(n=146)$, estudiantes universitarios, que solicitaron consulta en un centro de salud mental de la red asistencial pública $(n=66)$ y 
en la unidad de atención psicológica y salud mental para el universitario de la Universidad de Salamanca $(n=80)$. De ellos 96 eran mujeres $(65,8 \%)$ y 50 varones $(34,2 \%)$. La media de edad era de 23,8 años (desviación típica: 6,05). Todos los sujetos eran estudiantes universitarios, pero de ellos 76 estaban en el primer ciclo de estudios (52,1\%), 55 en el segundo ciclo $(36,3 \%)$ y 15 en cursos de doctorado o tercer ciclo $(11,6 \%)$. Los criterios de selección de la muestra eran que fuesen estudiantes universitarios y que fuese la primera vez que solicitaban consulta. Los criterios de exclusión eran estar en tratamiento psicofarmacológico y/o presentar sintomatología clara del área psicótica. Se pretendía obtener perfiles psicopatológicos de personas que presentaran diferentes síntomas y trastornos, con el objetivo de poder cubrir la mayor cantidad posible de categorías clínicas.

\section{Procedimiento}

A los sujetos no clínicos se les administró únicamente las escalas IAS de forma colectiva y en una única sesión de evaluación. La participación en el estudio era totalmente voluntaria, y se insistió en que las respuestas eran totalmente anónimas. El objetivo eran conseguir la mayor sinceridad y motivación hacia la tarea. En la Tabla 1 vemos los estadísticos descriptivos de las IAS de los sujetos no clínicos.

Los sujetos clínicos cumplimentaron el MCMI-II, SCL-90-R e IAS, siempre en este orden. La administración de los cuestionarios se hacía en la primera entrevista clínica, de forma individual, como parte de una evaluación rutinaria y durante una única sesión de evaluación. Siempre se solicitaba el permiso de la persona y se le garantizaban todos los aspectos relacionados con la confidencialidad de los datos. La administración de los cuestionarios duraba entre una hora y media y dos horas y los resultados eran utilizados posteriormente para la intervención clínica. En la Tabla 2 vemos los estadísticos descriptivos de los cuestionarios administrados a los sujetos clínicos.

\section{RESULTADOS Y DISCUSIÓN}

\section{Comparación entre sujetos clínicos} y no clínicos en las escalas IAS y en la puntuación de longitud del vector

Uno de nuestros objetivos es comprobar si hay diferencias entre los sujetos clínicos y no clínicos en cuanto a su estilo de conducta interpersonal autoinformada. Encontramos que en cuanto a las escalas IAS, la muestra de sujetos clínicos tiene

Tabla 1. Estadísticos descriptivos (sujetos no clínicos)

\begin{tabular}{llcc}
\hline Escalas IAS & & Media & DT \\
\hline PA & Seguro-dominante & 39,77 & 6,97 \\
BC & Arrogante-calculador & 28,55 & 7,19 \\
DE & Frío de ánimo & 18,96 & 7,04 \\
FG & Reservado-introvertido & 19,37 & 7,31 \\
HI & Inseguro-dependiente & 30,91 & 7,12 \\
JK & Ingenuo-modesto & 43,79 & 5,82 \\
LM & Cálido-afectuoso & 48,28 & 5,62 \\
NO & Gregario-extravertido & 49,42 & 6,21 \\
LNGV & Longitud del vector & 1,08 & 0,53 \\
\hline
\end{tabular}

Nota: IAS = Escalas de adjetivos Interpersonales. 
Tabla 2. Estadísticos descriptivos (sujetos no clínicos)

\begin{tabular}{|c|c|c|}
\hline Escalas IAS & Media & DT \\
\hline PA (Seguro-dominante) & 35,94 & 9,21 \\
\hline BC (Arrogante-calculador) & 26,98 & 7,64 \\
\hline DE (Frío de ánimo) & 20,93 & 7,96 \\
\hline FG (Reservado-introvertido) & 25,42 & 10,87 \\
\hline HI (Inseguro-dependiente) & 35,38 & 9,80 \\
\hline $\mathrm{JK}$ (Ingenuo-modesto) & 42,14 & 7,50 \\
\hline LM (Cálido-afectuoso) & 47,53 & 6,87 \\
\hline NO (Gregario-extravertido) & 44,28 & 9,69 \\
\hline \multicolumn{3}{|l|}{ MCMI-II TP } \\
\hline Esquizoide & 21,75 & 8,16 \\
\hline Fóbica (Evitativa) & 25,90 & 13,15 \\
\hline Dependiente & 29,73 & 9,40 \\
\hline Histriónica & 30,89 & 10,75 \\
\hline Narcisista & 34,80 & 10,3 \\
\hline Antisocial & 28,99 & 11,49 \\
\hline Agresivo-sádico & 33,43 & 12,08 \\
\hline Compulsiva & 33,93 & 9,31 \\
\hline Pasivo-agresiva & 35,92 & 13,40 \\
\hline Autodestructiva & 26,01 & 13,51 \\
\hline Esquizotípica & 21,20 & 12,54 \\
\hline Límite & 37,80 & 18,13 \\
\hline Paranoide & 27,26 & 10,95 \\
\hline \multicolumn{3}{|l|}{ MCMI-II SC } \\
\hline Ansiedad & 16,94 & 12,19 \\
\hline Histeriforme & 20,10 & 11,22 \\
\hline Hipomanía & 25,83 & 9,67 \\
\hline Neurosis depresiva & 25,50 & 16,88 \\
\hline Abuso de alcohol & 19,99 & 7,18 \\
\hline Abuso de drogas & 28,35 & 11,25 \\
\hline Pensamiento psicótico & 15,55 & 9,23 \\
\hline Depresión mayor & 19,36 & 13,52 \\
\hline Trastorno delirante & 12,83 & 7,55 \\
\hline \multicolumn{3}{|l|}{ SCL-90-R } \\
\hline Somatización & 16,75 & 12,19 \\
\hline Obsesivo-compulsiva & 13,98 & 7,99 \\
\hline Sensibilidad interpersonal & 12,54 & 8,04 \\
\hline Depresión & 20,65 & 11,57 \\
\hline Ansiedad & 13,37 & 7,99 \\
\hline Miedo-hostilidad & 5,40 & 5,32 \\
\hline Fobia & 4,89 & 4,91 \\
\hline Ideación paranoide & 6,94 & 5,21 \\
\hline Psicoticismo & 9,19 & 6,76 \\
\hline
\end{tabular}

Nota : IAS = Escalas de Adjetivos Interpersonales. MCMI-II TP = Trastornos de personalidad del Inventario Clínico Multiaxial de Millon. MCMI-II. SC = Síndromes clínicos del Inventario Clínico Multiaxial de Millon. SCL-90-R = Inventario de Síntomas de Derogatis.

puntuaciones medias superiores, estadísticamente significativas en las escalas $\mathrm{DE}$ (Frío de ánimo), FG (Reservado-introvertido), HI (Inseguro-dependiente) y en la puntuación de Longitud del vector. Mientras que los sujetos no clínicos tienen puntuaciones superiores, significativas estadísticamente en las escalas PA 
Tabla 3. Comparaciones entre los participantes clínicos $(n=146)$ y no clínicos $(n=140)$ en las puntuaciones medias de las Escalas IAS y LNV

\begin{tabular}{|c|c|c|c|c|c|}
\hline & Grupo & Media & DT & $t$ & $\begin{array}{c}\text { Sig. } \\
\text { bilateral }\end{array}$ \\
\hline \multirow[t]{2}{*}{$\overline{P A}$} & No clínico & 39,77 & 9,21 & 3,230 & $0,002^{\star \star}$ \\
\hline & Clínico & 35,94 & 9,21 & & \\
\hline \multirow[t]{2}{*}{$\mathrm{BC}$} & No clínico & 28,55 & 7,19 & 1,501 & 0,136 \\
\hline & Clínico & 26,98 & 7,64 & & \\
\hline \multirow[t]{2}{*}{$\overline{\mathrm{DE}}$} & No clínico & 18,96 & 7,04 & $-2,219$ & 0,029 \\
\hline & Clínico & 20,93 & 7,96 & & \\
\hline \multirow[t]{2}{*}{ FG } & No clínico & 19,37 & 7,31 & $-4,763$ & $0,000^{\star \star \star \star}$ \\
\hline & Clínico & 25,42 & 10,87 & & \\
\hline \multirow[t]{2}{*}{ HI } & No clínico & 30,91 & 7,12 & $-4,329$ & $0,000^{\star \star \star}$ \\
\hline & Clínico & 35,38 & 9,80 & & \\
\hline \multirow[t]{2}{*}{$\mathrm{JK}$} & No clínico & 43,79 & 5,82 & 2,123 & $0,036^{\star}$ \\
\hline & Clínico & 42,14 & 7,50 & & \\
\hline \multirow[t]{2}{*}{ LM } & No clínico & 48,28 & 5,62 & 1,029 & 0,30 \\
\hline & Clínico & 47,53 & 6,87 & & \\
\hline \multirow[t]{2}{*}{ NO } & No clínico & 49,42 & 6,21 & 4,449 & $0,000^{* \star *}$ \\
\hline & Clínico & 44,28 & 9,69 & & \\
\hline \multirow[t]{2}{*}{$\overline{\mathrm{LNV}}$} & No clínico & 1,084 & 0,533 & $-3,314$ & 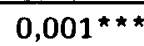 \\
\hline & Clínico & 1,39 & 0,79 & & \\
\hline
\end{tabular}

${ }^{\star} p \leq 0,05 ;{ }^{\star \star} p \leq 0,01 ;{ }^{\star \star \star} p \leq 0,001$

(Seguro-dominante), JK (Ingenuo-modesto) y NO (Gregario-extravertido) (ver Tabla 3).

Estos resultados indican que los sujetos de la muestra clínica se describen a sí mismos en su relaciones interpersonales como más fríos de ánimo, introvertidos e inseguros, en comparación con los sujetos de la muestra no clínica, que se describen a sí mismos como más seguros, ingenuos y extravertidos. En general los sujetos de la muestra clínica tienen puntuaciones más elevadas en las escalas del cuadrante inferior izquierdo, mientras que los sujetos de la muestra no clínica lo hacen en el cuadrante superior e inferior derecho, la parte afiliativa del círculo, excluyendo el octante LM (Cálido-agradable) en el que las diferencias no son significativas, aunque la tendencia de una puntuación superior se mantiene.
Respecto a las puntuaciones resumen de las IAS encontramos la puntuación de Longitud de vector (LongV) como diferente significativamente en las muestras de sujetos clínicos y no clínicos, en el sentido de una puntuación media superior estadísticamente significativa en la muestra de sujetos clínicos. Este resultado puede indicarnos que en nuestra muestra de sujetos clínicos aparecen patrones de conducta interpersonal que son más intensos, exagerados, inflexibles y disfuncionales, algo ya indicado por Wiggins (1996) y que confirmamos con estos resultados.

Para ver las implicaciones psicopatológicas de esta puntuación seleccionamos dos subgrupos entre los sujetos clínicos con puntuaciones extremas en LongV: sujetos con puntuaciones superiores al percentil $75\left(\mathrm{P}_{75}: 1,74 ; n=36\right)$ y sujetos con puntuaciones inferiores al percentil 
Tabla 4. Comparaciones entre medias (escalas del MCMI-II y del SCL-90-R) entre los sujetos clínicos con puntuaciones de Longitud del vector inferiores al percentil $25(n=36)$ y superiores al percentil $75(n=39)$

\begin{tabular}{|c|c|c|c|c|c|}
\hline & Percentil & Media & DT & $t$ & $\begin{array}{c}\text { Sig. } \\
\text { bilateral }\end{array}$ \\
\hline Z/MCMI2 & $\begin{array}{l}<25 \\
>75\end{array}$ & $\begin{array}{l}13,17 \\
20,08\end{array}$ & $\begin{array}{r}8,84 \\
10,67\end{array}$ & $-3,039$ & $0,003^{\star *}$ \\
\hline 1/MCMI2 & $\begin{array}{l}<25 \\
>75\end{array}$ & $\begin{array}{l}19,14 \\
26,95\end{array}$ & $\begin{array}{l}5,59 \\
8,92\end{array}$ & $-4,501$ & $0,000^{\star \star \star}$ \\
\hline 2/MCMI2 & $\begin{array}{l}<25 \\
>75\end{array}$ & $\begin{array}{l}22,14 \\
33,95\end{array}$ & $\begin{array}{l}11,07 \\
14,35\end{array}$ & $-3,966$ & $0,000^{* * *}$ \\
\hline 4/MCMI2 & $\begin{array}{l}<25 \\
>75\end{array}$ & $\begin{array}{l}32,22 \\
27,02\end{array}$ & $\begin{array}{r}8,77 \\
12,17\end{array}$ & 2,106 & $0,039^{\star}$ \\
\hline 8B/MCMI2 & $\begin{array}{l}<25 \\
>75\end{array}$ & $\begin{array}{l}23,92 \\
30,54\end{array}$ & $\begin{array}{l}12,02 \\
15,14\end{array}$ & $-2,087$ & $0,040^{\star}$ \\
\hline S/MCMI2 & $\begin{array}{l}<25 \\
>75\end{array}$ & $\begin{array}{l}17,72 \\
\mathbf{2 8 , 7 4}\end{array}$ & $\begin{array}{l}10,05 \\
13,23\end{array}$ & $-4,038$ & $0,000^{\star \star \star}$ \\
\hline C/MCMI2 & $\begin{array}{l}<25 \\
>75\end{array}$ & $\begin{array}{l}34,64 \\
44,28\end{array}$ & $\begin{array}{l}15,64 \\
18,18\end{array}$ & $-2,452$ & $0,017^{*}$ \\
\hline A/MCMI2 & $\begin{array}{l}<25 \\
>75\end{array}$ & $\begin{array}{l}15,17 \\
21,28\end{array}$ & $\begin{array}{l}11,34 \\
12,46\end{array}$ & $-2,217$ & $0,030^{*}$ \\
\hline N/MCMI2 & $\begin{array}{l}<25 \\
>75\end{array}$ & $\begin{array}{l}27,78 \\
23,28\end{array}$ & $\begin{array}{l}8,19 \\
9,99\end{array}$ & 2,121 & $0,037^{\star}$ \\
\hline $\mathrm{D} / \mathrm{MCMI} 2$ & $\begin{array}{l}<25 \\
>75\end{array}$ & $\begin{array}{l}20,53 \\
32,72\end{array}$ & $\begin{array}{l}13,56 \\
17,77\end{array}$ & $-3,319$ & $0,001^{* \star \star}$ \\
\hline SS/MCMI2 & $\begin{array}{l}<25 \\
>75\end{array}$ & $\begin{array}{l}13,72 \\
19,15\end{array}$ & $\begin{array}{r}7,1 \\
9,25\end{array}$ & $-2,836$ & $0,006^{* *}$ \\
\hline $\mathrm{CC} / \mathrm{MCMI} 2$ & $\begin{array}{l}<25 \\
>75\end{array}$ & $\begin{array}{l}15,61 \\
24,56\end{array}$ & $\begin{array}{l}11,37 \\
13,24\end{array}$ & $-3,130$ & $0,003^{\star *}$ \\
\hline 3/SCL90R & $\begin{array}{l}<25 \\
>75\end{array}$ & $\begin{array}{l}11,55 \\
15,54\end{array}$ & $\begin{array}{l}7,51 \\
8,40\end{array}$ & $-2,157$ & $0,034^{*}$ \\
\hline $6 /$ SCL90R $<25$ & $\begin{array}{l}4,33 \\
>75\end{array}$ & $\begin{array}{l}3,64 \\
7,10\end{array}$ & $\begin{array}{r}-2,297 \\
6,33\end{array}$ & $0,024^{*}$ & \\
\hline
\end{tabular}

${ }^{\star} p \geq 0,05 ;{ }^{\star \star} p \geq 0,01 ;{ }^{\star \star \star} p \geq 0,001$.

$25\left(\mathrm{P}_{25}: 0,81 ; n=35\right)$, y analizamos si existen diferencias significativas on las escalas del MCMI-II y SCL-90-R entre ambas submuestras de sujetos clínicos.

En la Tabla 4 vemos los resultados significativos $^{1}$ de la comparación de medias mediante la prueba $t$ de Student para muestras independientes de las escalas de los cuestionarios MCMI-II y SCL-90-R.

1 Incluimos únicamente los resultados significativos estadísticamente; si se desean los resultados completos pueden ser solicitados a los autores.
Para las variables del MCMI-II encontramos diferencias significativas en varias escalas. En concreto, puntuaciones medias superiores estadísticamente significativas en el subgrupo de sujetos con puntuaciones de LongV inferiores al percentil 25 en las escalas de Deseabilidad (Y), Personalidad histriónica (4) e Hipomanía (N), y puntuaciones media superiores estadísticamente significativas en el subgrupo de sujetos con puntuaciones de LongV superiores al percentil 75 en las escalas de Alteración 
(Z), Personalidad esquizoide (1), Fóbica (2), Autodestructiva (8B), Esquizotípica (S) y Límite (C), y en las escalas clínicas de Ansiedad (A), Neurosis depresiva (D), Pensamiento psicótico (SS) y Depresión mayor (CC).

En el SCL-90-R encontramos puntuaciones medias superiores estadísticamente significativas en el subgrupo de sujetos clínicos con puntuaciones de LongV superiores al Percentil 75 en las escalas de Sensibilidad interpersonal (3) y Depresión (6).

En general los sujetos clínicos con puntuaciones de Longitud de vector superiores a 1,74 obtienen puntuaciones superiores en 11 escalas psicopatológicas de los cuestionarios MCMI-II y SCL90-R, mientras los sujetos clínicos con puntuaciones de Longitud de vector inferiores a 0,81 tienen puntuaciones superiores en la escala de deseabilidad y en dos escalas psicopatológicas del MCMI-II. Según estos resultados podemos decir que los sujetos clínicos con puntuaciones inferiores al percentil 25 en LONGV intentan dar una imagen socialmente deseable de si mismos, causar buena impresión y parecer mentalmente sanos. Por otro lado la aparición de puntuaciones más elevadas que presentan en las escalas de trastorno histriónico de la personalidad e hipomanía, pueda tener que ver con una menor capacidad de percepción de sus propias dificultades, así como con desinterés por la introspección, que puede ser bien debido a su inconstancia y superficialidad (trastorno histriónico de la personalidad) o a su sobreactividad, impulsividad y elevada distraibilidad (hipomanía).

En cuanto a los sujetos clínicos con puntuaciones de longitud de vector superiores a 1,74 , presentan puntuaciones medias superiores en la escala de alteración, lo que podría indicar que tienen tendencia a degradarse, a acentuar su angustia psicológica y exhibir su vulne- rabilidad emocional, haciendo lo posible por dar una mala impresión y exagerar sus verdaderas dificultades sociales y emocionales. En cuanto a las escalas psicopatológicas encontramos puntuaciones medias superiores en las escalas de trastornos de la personalidad Esquizoide, Fóbica, Autodestructiva, Esquizotípica y Límite, y en las escalas clínicas de Ansiedad, Neurosis depresiva, Pensamiento psicótico y Depresión mayor del MCMIII y en las Sensibilidad interpersonal y Depresión del SCL-90-R. Debido al número y diversidad de las escalas psicopatológicas es difícil concluir alguna característica particular de estos participantes, quizás lo único que podemos decir es que presentan puntuaciones superiores en un número mayor de escalas psicopatológicas, en comparación con el grupo de puntuaciones inferiores en LongV, siendo alguna de ellas graves, como los trastornos de la personalidad esquizotípico y límite (Millon, 1998), y en torno a características de aislamiento social y sintomatología ansioso-depresiva. Sí debemos destacar la puntuación media superior en la escala de Sensibilidad interpersonal del SCL-90-R, que tiene que ver con sentimientos de incapacidad personal e inferioridad en comparación con otras personas, así como gran malestar durante la interacción interpersonal, con sentimientos de timidez y expectativas negativas en la comunicación interpersonal.

En resumen, los sujetos clínicos con puntuaciones de Longitud de vector altas obtienen puntuaciones superiores en un número mayor de escalas psicopatológicas, que los sujetos clínicos con puntuaciones de LongV inferiores. Según estos resultados podemos decir que en población clínica la puntuación de Longitud del vector es un indicador de amplitud psicopatológica general, pero no de ninguna categoría psicopatológica específica. 
Tabla 5. Coeficientes de correlación de Pearson entre la Longitud del vector y las variables Deseabilidad, Alteración, Índice Global de Gravedad, Total de Síntomas positivos e Índice de Malestar $(n=146)$

\begin{tabular}{lccccc}
\hline & Y/MCMI2 & Z/MCMI2 & IGS & TPS & PSDI \\
\hline LNV & $-0,274^{\star \star \star}$ & $0,249^{\star \star}$ & $0,181^{*}$ & 0,097 & $0,192^{\star}$ \\
\hline
\end{tabular}

${ }^{\star} p \leq 0,05^{\star \star \star} ; p \leq 0,01 ;{ }^{\star \star \star} p \leq 0,001$.

El cálculo de las correlaciones de la puntuación de LongV con las puntuaciones de Alteración ( $\mathrm{Z}$ ) y Deseabilidad (Y) del MCMI-II y las puntuaciones Îndice de Malestar (PSDI), Índice Global de Gravedad (IGS) y el Total de Síntomas Positivos (TPS), nos da mas indicadores del significado e importancia de esta puntuación (Ver Tabla 5).

Una puntuación elevada en LongV es un indicador de que la persona evaluada presentará probablemente tendencia a denigrarse y degradarse a sí misma, acentuar su angustia patológica y exhibir su vulnerabilidad emocional, y no mostrará interés por causar buena impresión, aparecer mentalmente sana o socialmente virtuosa. Podemos considerar la puntuación en Longitud del vector como un indicador del nivel o profundidad del malestar, de la respuesta psicológica al trastorno y de una tendencia al aumento del malestar sintomático del que nos informa el sujeto, en sujetos clínicos.

Estos resultados muestran la puntuación de longitud del vector como una medida de desviación psicopatológica en sujetos clínicos, con lo que no confirmamos lo obtenido por Wiggins et al. (1988) para quienes la puntuación de LongV no es en sí misma una medida genérica de desviación o psicopatología, aunque hemos de decir que los resultados de Wiggins et al. (1988) fueron obtenidos con sujetos no clínicos.

\section{Análisis de los perfiles interpersonales}

En el grupo de sujetos clínicos, que es la que vamos a analizar, encontramos 51 perfiles irregulares, un $34,93 \%$, de un total de 146 perfiles. Para analizar su significado clínico comparamos las puntuaciones en los cuestionarios MCMI-II y SCL-90-R de los sujetos clínicos con perfiles regulares con las puntuaciones de los sujetos clínicos con perfiles irregulares.

En la Tabla 6 parecen las escalas psicopatológicas del MCMI-II y SCL-90-R en las que las diferencias de medias son significativas estadísticamente. Según la prueba de comparación de medias encontramos diferencias estadísticamente significativas al $5 \%$ en 3 escalas del MCMI-II, en concreto en las escalas de trastorno de la personalidad Narcisista y Paranoide y la escala de Trastorno delirante, en el sentido de una media superior en las tres escalas para la muestra de sujetos con perfiles irregulares. Un resultado importante es que dentro de las escalas de validez del MCMI-II no hay diferencias significativas en ambos grupos de sujetos en las escalas de sinceridad y deseabilidad, con lo cual estos resultados probablemente no son debidos a la ausencia de sinceridad en la respuesta de los sujetos con perfiles irregulares, como postulaba Wiggins (1996).

Según estos resultados los sujetos clínicos con perfiles irregulares serían personas que se burlan de las reglas establecidas, sienten que pueden hacer lo que desean en cualquier momento y que de algún modo son "especiales». Por otro lado muestran cierta resistencia a las demandas externas, buscando motivos ocultos y desconfiando de los demás. Podría existir una comprensión 
distorsionada de la tarea o bien del significado de algunos adjetivos, así como algún trastorno del pensamiento y sospecha hacia la tarea o la situación de evaluación.

\section{DISCUSIÓN Y CONCLUSIONES}

Con este trabajo pretendemos poner de manifiesto las características y utilidad clínica del modelo interpersonal de Wiggins y de su instrumento de evaluación, las Escalas de Adjetivos Interpersonales, mediante el estudio del estilo de conducta interpersonal en sujetos clínicos y su comparación con sujetos no clínicos, y el análisis de la importancia clínica de los perfiles interpersonales obtenidos mediante las IAS y su significado psicopatológico.

La utilidad clínica del circumplex interpersonal de Wiggins, se manifiesta fundamentalmente a través del perfil interpersonal que nos ofrecen las IAS y esta utilidad es evidente en dos aspectos: las características del estilo interpersonal de los sujetos clínicos y las diferencias, si las hay, con los sujetos no clínicos y la utilidad para la planificación de objetivos de la intervención psicológica y la evaluación de la eficacia de la intervención.

Con respecto al primer aspecto, objetivo de este trabajo, las Escalas de Adjeti- vos Interpersonales muestran diferencias en el estilo interpersonal entre sujetos clínicos y no clínicos, de forma que se describen a sí mismos y en sus relaciones interpersonales con estilos muy diferentes e incluso opuestos. En los sujetos clínicos estos estilos interpersonales presentan características asociadas a un estilo frío e introvertido, y son perfiles interpersonales más rígidos e inflexibles, es decir, les cuesta más adaptarlos a las demandas de la situación, al contrario de lo que sucede con los sujetos no clínicos, que obtienen perfiles interpersonales mas flexibles y adaptativos y con un contenido interpersonal cálido y extrovertido. En general, si administramos las IAS a una persona y obtiene un perfil con características interpersonales pertenecientes al cuadrante inferior izquierdo y con una longitud del vector elevada podría indicarnos la existencia de dificultades relacionales que podrían manifestarse clínicamente. Numerosos trabajos se ha encargado de estudiar estas manifestaciones, en concreto las correspondencias de los octantes IAS con los trastornos de personalidad y síndromes clínicos (p.ej., Felipe y Ávila, en prensa) y ponen de manifiesto la necesidad de una evaluación dimensional del contenido interpersonal asociado a cada categoría de trastorno psicopatológico para lograr una mayor comprensión de su contenido interpersonal.

Tabla 6. Comparaciones entre medias (escalas del MCMI-II y del SCL-90-R) entre los sujetos con perfiles regulares $(n=95)$ y perfiles irregulares $(n=51)$

\begin{tabular}{llrrr}
\hline & Perfil & Media & $D T$ & $t$ \\
\hline Z/MCMI2 & Irregular & 37,14 & 9,64 & 2,026 \\
& Regular & 33,55 & 10,45 & 2,045 \\
\hline P/MCMI2 & Irregular & 29,74 & 11,11 & 2,041 \\
& Regular & 25,93 & 10,56 & 7,68 \\
\hline PP/MCMI2 & Irregular & 14,55 & 7,25 & \\
& Regular & 11,93 & & \\
\hline
\end{tabular}

${ }^{*} p \leq 0,05$ (significación bilateral). 
Respecto a los perfiles irregulares, los resultados que hemos obtenido ponen de manifiesto lo que Wiggins indicaba, la necesidad de considerarlos con cautela. Nuestros resultados indican que los sujetos clínicos que presentan este tipo de perfiles son personas con unas características de personalidad que les lleva a poner de manifiesto las diferencias con los demás y el sentirse como personas "especiales». Son personas desconfiadas y que buscan motivos ocultos en los demás, y estas características las hacen extensibles a situaciones de evaluación psicológica. Por otro lado las puntuaciones de los sujetos clínicos con perfiles regulares e irregulares en escalas de Sinceridad y Deseabilidad del MCMI-II son similares (no hay diferencias significativas en las puntuaciones, ver Tabla 6) con lo cual debemos concluir que estos resultados no son debidos a que los sujetos mientan o manipulen las respuestas, si no que ha sido sinceros y francos y se han expresado libremente sin intentar dar una imagen socialmente deseable de si mismos. En general, si nos encontramos con un perfil irregular debemos tener en cuenta estas características y debemos realizar una evaluación más profunda respecto a otros aspectos psicopatológicos y de la personalidad que puedan aclararnos el significado de este estilo interpersonal dentro de la historia de vida y clínica de la persona.

Por otro lado los perfiles interpersonales obtenidos con las IAS servirían como marcadores de las áreas interpersonales desadaptativas a partir de las cuales plantear objetivos para la intervención, tanto para flexibilizar el estilo interpersonal como para intentar desarrollar estilos de comportamiento relacional mas adaptativos. Las psicoterapias interpersonales han puesto de manifiesto estos objetivos de intervención y la utilidad de la evaluación del estilo interpersonal para la planificación de objetivos psicoterapéuticos (Anchin y Kiesler, 1982).
Nos encontramos por tanto ante un instrumento que cuenta con un importante apoyo teórico y empírico que permite su uso tanto en contextos clínicos como educativos y organizacionales, proporcionándonos en todos los casos una evaluación fiable y útil para fines de orientación y de intervención clínica. Es claro que dentro de esta utilidad el análisis del perfil interpersonal obtenido así como de la puntuación de Longitud del vector son dos de los aspectos a tener en cuenta si pretendemos aprovechar las posibilidades de evaluación y diagnóstico de las Escalas de Adjetivos Interpersonales (IAS).

\section{REFERENCIAS BIBLIOGRÁFICAS}

Alden, L.E., Wiggins, J.S. y Pincus, A.L. (1990). Construction of circumplex scales for the Inventory of Interpersonal Problems. Journal of Personality Assessment, 55, 521-535.

Allport, G.W. y Odberg, H.S. (1936). Traitnames: A psycho-lexical study (Número especial). Psychological Monographs, 47.

Anchin, J.C. y Kiesler, D. (1982). Handbook of interpersonal psychotherapy. New York: Pergamon Press.

Beringer, V.W. y DeSoto, C. (1985). Cognitive representations of social stereotipes. European Journal of Social Psychology, 15, 189-211.

Buss, D.M. y Craik, K.H. (1985). Why not measure that trait? Alternative criteria for identifying dispositions. Journal of Personality and Social Psychology, 48, 934-946.

Carson, R.C. (1969). Interaction concepts in personality. Chicago: Aldine.

Cheek, J.M. y Hogan, R. (1982). Self-concepts, self-representation and moral judments. En J. Suls y A.G. Greenwald (Eds.), Psychological perspectives on the self (Vol. 2, pp. 249-273). Hillsdales, NJ: Erlbaum.

Dempsey, M., Lamon, S., Suderland, P. y DiGiuseppe, R. (1994). Effects of client personality on dominance and affiliation during sessions of rational-emotive psychotherapy. Journal of Rational Emotive and Cognitive Behavior Therapy, 12, 237-256. 
Derogatis, L.R. (1983). SCL-90-R. Administration, scoring and procedures Manual-II (2nd ed.). Baltimore, MD: Clinical Psychometric Research.

Felipe, E. y Ávila-Espada, A. (1999). Perfiles diferenciales interpersonales en personas con lesión medular traumática. Revista Mapfre Medicina, 3, 159-157.

Felipe, E. y Ávila-Espada, A. (2001). Clinicaldiagnostic value of Interpersonal Adjetive Scales (IAS). Journal of Personality Assessment (En prensa).

Fichten, C.S. y Amsel, R. (1986). Trait attributions about college students with physical disability: Circumplex analyses and methodological issues. Journal of Applied Social Psychology, 16, 411-427.

Foa, U.G. y Foa, E.B. (1974). Societal structures of the mind. Springfield, IL: Charles C. Thomas.

Freedman, M.B., Leary, T., Ossorio, A.G. y Coffey, H.S. (1951). The interpersonal dimension of personality. Journal of Personality, 20, 143-161.

Goffman, E. (1959). The presentation of self in everyday life. Garden City, NY: Doubleday Anchor Books.

Goldberg, L.R. (1977/Agosto). Language and personality: Developing a taxonomy of traitdescriptive terms. Invited address to the Division of Evaluation and Measurement at the $86^{\text {th }}$. Annual Convention of the American Psychological Association, San Francisco.

Goldberg, L. R. (1982). From Ace to Zombie: Some explorations in the language of personality. In C. D. Spielberger \& J. N. Butcher (Eds.), Advances in personality assessment: Vol. 1 (pp. 203-234). Hillsdale, N.J.: Erlbaum.

González de Rivera, J.L., Derogatis, L.R., De las Cuevas, C., Gracia-Marco, R., Rodriguez-Pulido, F., Henry-Benitez, M., y Monterrey, A.L. (1989). The spanish version of the SCL-90-R. Normative data in general population. Baltimore, MD: Clinical Psychometric Research.

Guttman, L. (1984). A new approach to factor analysis: The radex. In P.F. Lazarsfeld (Ed.), Mathematical thinking in the social sciences (pp.258-348). Chicago: Free Press.

Kelley, H.H. (1983). The situacional origins of human tendencies: A further reason for the formal analysis of structures. Personality and Social Psychology Bulletin, 9, 8-30.

Kiesler, D.J. (1982). Interpersonal theory for personality and psychotherapy. En J.C. Anchin y D.J. Kiesler (Eds.), Handbook of interpersonal psychotherapy (pp. 3-24). Elmsford, NY: Pergamon Press.

Leary, T. (1957). Interpersonal diagnosis of personality: A functional theory and methodology for personality evaluation. New York: Ronald Press.

Madison, J.K. (1997). Interpersonal assessment and therapy of eating disorders: A clinical application of a circumplex model. En R. Plutchik y H.R. Conte (Eds.), Circumplex models of personality and emotions (pp. 431-447). Washington, DC: American Psychological Association.

Millon, T. (1987). Millon Clinical Multiaxial Inventory (MCMI-II). Minneapolis, MN: National Computer Systems.

Millon, T. (1998). MCMI-II. Inventario clínico multiaxial de Millon-II. Manual. Madrid: TEA Ediciones.

Norman, W.T. (1967). 2800 personality trait descriptors: Normative operating characteristics for a university population. Manuscrito no publicado. Universidad de Michigan.

Pincus, A.L. y Wiggins, J.S. (1990). Interpersonal problems and conceptions of personality disorders. Journal of Personality Disorders, 4, 340-352.

Romer, D. y Revelle, W. (1985). Personality traits: Fact or fiction? Journal of Personality and Social Psychology, 47, 1028-1042.

Sullivan, H.S. (1953). The interpersonal theory of Psychiatry. New York: Norton. (Trad. Cast. Buenos Aires: Hormé).

Trapnell, P.D. y Wiggins, J.S. (1990). Extension of the Interpersonal Adjetive Scales to include the Big Five dimensions of personality. Journal of Personality and Social Psychology, 59, 781-790.

Wiggins, J.S. (1979). A psychological taxonomy of trait-descriptive terms: The interpersonal domain. Journal of Personality and Social Psychology, 37, 395-412.

Wiggins, J.S. (1980). Circumplex models of interpersonal behavior. En L. Wheeler (Ed.), Review of Personality and Social Psychology (Vol. 1, pp. 265-293). Beverly Hills: Sage. 
Wiggins, J.S. (1982). Circumplex models of interpersonal behavior in clinical psychology. En P.C. Kendall y J.N. Butcher (Eds.), Handbook of research methods in clinical psychology (pp. 1 83-221). New York: Wiley.

Wiggins, J.S. (1996). Escala de Adjetivos Interpersonales (IAS) Manual profesional. Madrid: TEA Ediciones.

Wiggins, J.S., y Broughton, R. (1991). A geometric taxonomy of personality scales. European Journal of Personality, 5, 343-365.

Wiggins, J.S. y Pincus, A.L. (1989). Conceptions of personality disorders and dimensions of personality. Psychological Assessment, 1, 305-316.
Wiggins, J.S. y Pincus, A.L. (1992). Personality: Structure and assessment. En M.R. Rosenzweig y L.W. Porter (Eds.), Annual Review of Psychology (Vol. 43, pp. 473504). Palo Alto: Annual Reviews.

Wiggins, J.S. y Trapnell, P.D. (1996). A dyadic-interactional perspective on the Fivefactor model. En J.S. Wiggins (Ed.), The Five-factor Model of Personality: Theoretical perspectives (pp. 88-162). New York: Guilford Press.

Wiggins, J.S., Trapnell, P.D. y Phillips, N. (1988). Psychometric and Geometric Characteristics of the Revised Interpersonal Adjetive Scales (IAS-R). Multivariate Behavioral Research, 23, 517-530. 